\title{
Real-time dynamics of Auger wave packets and decays in ultrafast charge migration processes
}

\author{
F. Covito, ${ }^{1}$ E. Perfetto, ${ }^{2,3}$ A. Rubio, ${ }^{1,4,5}$ and G. Stefanucci ${ }^{3,6}$ \\ ${ }^{1}$ Max Planck Institute for the Structure and Dynamics of Matter and Center for Free-Electron Laser Science, \\ Luruper Chaussee 149, 22761 Hamburg, Germany \\ ${ }^{2}$ CNR-ISM, Division of Ultrafast Processes in Materials (FLASHit), Area della ricerca di Roma 1, Monterotondo Scalo, Italy \\ ${ }^{3}$ Dipartimento di Fisica, Università di Roma Tor Vergata, Via della Ricerca Scientifica, 00133 Rome, Italy \\ ${ }^{4}$ Center for Computational Quantum Physics (CCQ), The Flatiron Institute, 162 Fifth avenue, New York, New York 10010, USA \\ ${ }^{5}$ Nano-Bio Spectroscopy Group, Universidad del País Vasco, 20018 San Sebastian, Spain \\ ${ }^{6}$ INFN, Sezione di Roma Tor Vergata, Via della Ricerca Scientifica 1, 00133 Roma, Italy
}

(Received 15 December 2017; revised manuscript received 27 March 2018; published 6 June 2018)

\begin{abstract}
The Auger decay is a relevant recombination channel during the first few femtoseconds of molecular targets impinged by attosecond XUV or soft x-ray pulses. Including this mechanism in time-dependent simulations of charge-migration processes is a difficult task, and Auger scatterings are often ignored altogether. In this work we present an advance of the current state-of-the-art by putting forward a real-time approach based on nonequilibrium Green's functions suitable for first-principles calculations of molecules with tens of active electrons. To demonstrate the accuracy of the method we report comparisons against accurate grid simulations of one-dimensional systems. We also predict a highly asymmetric profile of the Auger wave packet, with a long tail exhibiting ripples temporally spaced by the inverse of the Auger energy.
\end{abstract}

DOI: 10.1103/PhysRevA.97.061401

The subfemtosecond dynamics of the hole density created by an ionizing attosecond XUV or soft X-ray pulse precedes any nuclear rearrangement and dictates the relaxation pathways of the underlying molecular structure [1,2]. This ultrafast charge oscillation, also referred to as ultrafast charge migration (UCM), is driven exclusively by electronic correlations up to a few femtoseconds [3-7]. At these time scales the Auger scattering is the only possible energy-dissipation mechanism and, in addition to shake-up and polarization effects [8], a relevant recombination channel.

Recent advances in pump-probe spectroscopy have made it possible to follow the Auger decay in atomic targets [2,9-12]. Accurate measurements have been performed and successfully interpreted in terms of transitions between excited cationic states. The theory behind these experiments shows that the Auger electron is a "courier" of the complex dynamics occurring in the parent cation [13-15]. Unfortunately, ab initio analysis relying on many-electron eigenfunctions and eigenvalues are possible for single atoms but soon become prohibitive for larger systems. In fact, first-principles approaches that include Auger scatterings in the UCM dynamics of molecules have not yet been developed.

Time-dependent density functional theory [16-18] (TDDFT) is the method of choice for large-scale simulations. However, the vast majority of TDDFT calculations are performed using an adiabatic exchange-correlation (xc) potential, i.e., a functional of the instantaneous density. As shown in Ref. [19], adiabatic approximations are unable to capture the Auger effect [20]. Learning how to include memory effects in the xc functional is a major line of research to which the present work could provide new insights.

In this Rapid Communication we present a first-principles real-time nonequilibrium Green's function [21,22] (NEGF) approach which incorporates Auger scatterings in the UCM dynamics of molecules hit by attosecond pulses. In analogy with the NEGF formulation of quantum transport where the dynamics of electrons in the junction is simulated without dealing explicitly with the electrons in the leads [23-25], we close the NEGF equations on the molecule and deal only partially with the degrees of freedom of the Auger electrons. The computational effort changes slightly with respect to previous NEGF implementations [26-28], thereby making it possible to simulate the UCM of molecules with tens of active electrons.

We demonstrate that the approach well captures qualitative and quantitative aspects of the Auger physics through comparisons against real-time simulations of one-dimensional (1D) atoms on a grid. The Auger wave packet can, in principle, be reconstructed from NEGF through a postprocessing procedure. For three-dimensional (3D) molecules such procedure is numerically (too) demanding but for the considered 1D atom the calculation is doable and the agreement with the full-grid results is again satisfactory. Interestingly, we highlight a universal feature of the asymmetric Auger wave packet, namely, a long tail with superimposed ripples temporally spaced by the inverse of the Auger energy.

Method. We consider a finite system (an atom or molecule) with single-particle Hartree-Fock (HF) basis $\varphi_{i}(\mathbf{r})$ for bound electrons and $\varphi_{\mu}(\mathbf{r})$ for electrons in the continuum (for simplicity we work with spin-degenerate systems). Let $\hat{c}_{i \sigma}\left(\hat{c}_{\mu \sigma}\right)$ be the annihilation operator for an electron on $\varphi_{i}\left(\varphi_{\mu}\right)$ with spin $\sigma$. In the absence of external fields the total Hamiltonian

$$
\hat{H}^{\mathrm{eq}}=\hat{H}_{\text {bound }}+\hat{H}_{\text {Auger }}+\hat{H}_{\text {cont }}
$$


(a)

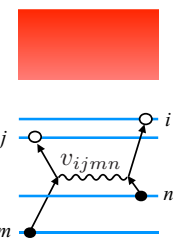

intra-molecular scattering

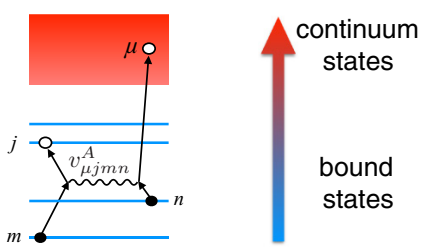

Auger scattering

(b)

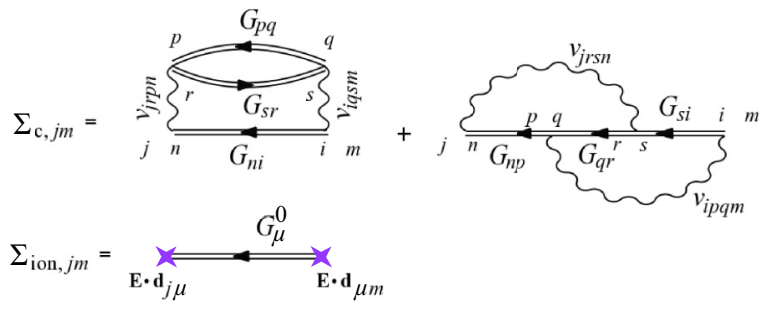

FIG. 1. (a) Schematic illustration of intramolecular (left) and Auger (right) scattering. (b) Correlation self-energy in the 2B approximation (top) and ionization self-energy (bottom).

is the sum of the bound-electrons Hamiltonian $\hat{H}_{\text {bound }}=$ $\sum_{\sigma} h_{i j} \hat{c}_{i \sigma}^{\dagger} \hat{c}_{j \sigma}+\frac{1}{2} \sum_{\substack{i j m n \\ \sigma \sigma^{\prime}}} v_{i j m n} \hat{c}_{i \sigma}^{\dagger} \hat{c}_{j \sigma^{\prime}}^{\dagger} \hat{c}_{m \sigma^{\prime}} \hat{c}_{n \sigma}$, the Auger interaction $\hat{H}_{\text {Auger }}=\sum_{i j m \mu} v_{i j m \mu}^{A}\left(\hat{c}_{i \sigma}^{\dagger} \hat{c}_{j \sigma^{\prime}}^{\dagger} \hat{c}_{m \sigma^{\prime}} \hat{c}_{\mu \sigma}+\right.$ H.c.), and a free-continuum part $\hat{H}_{\text {cont }}=\sum_{\mu \sigma} \epsilon_{\mu} \hat{c}_{\mu \sigma}^{\dagger} \hat{c}_{\mu \sigma}$. Here $h_{i j}$ are the one-electron integrals, $\epsilon_{\mu}$ are the continuum single-particle energies, and $v_{i j m n}\left(v_{i j m \mu}^{A}\right)$ are the four-index Coulomb integrals responsible for intramolecular (Auger) scatterings [see Fig. 1(a)].

The system is perturbed either by the sudden removal of a bound electron or by an external laser field. In the dipole approximation the laser-system interaction reads

$$
\hat{H}^{\mathbf{E}}(t)=\hat{H}_{\text {bound }}^{\mathbf{E}}(t)+\hat{H}_{\text {ion }}^{\mathbf{E}}(t),
$$

where $\hat{H}_{\text {bound }}^{\mathbf{E}}(t)=\mathbf{E}(t) \cdot \sum_{i} \mathbf{d}_{i j} \hat{c}_{i \sigma}^{\dagger} \hat{c}_{j \sigma}$ describes intramolecular transitions, whereas $\hat{H}_{\text {ion }}^{\mathbf{E}}(t)=\mathbf{E}(t) \cdot \sum_{i \mu}\left(\mathbf{d}_{i \mu} \hat{c}_{i \sigma}^{\dagger} \hat{c}_{\mu \sigma}+\right.$ H.c.) is responsible for ionization. The vector $\mathbf{d}_{i j}\left(\mathbf{d}_{i \mu}\right)$ is the matrix element of the dipole operator between states $\varphi_{i}$ and $\varphi_{j}\left(\varphi_{\mu}\right)$. In Eqs. (1) and (2) we are discarding the off-diagonal elements $h_{i \mu}, h_{\mu \mu^{\prime}}$, and $\mathbf{d}_{\mu \mu^{\prime}}$ as well as all Coulomb integrals with two or more indices in the continuum. We anticipate that this simplification only marginally affects the results presented below.

The electron dynamics is simulated using NEGF. Without Auger scatterings the equation of motion for the oneparticle density matrix $\rho_{i j}(t)=\left\langle\hat{c}_{j \sigma}^{\dagger}(t) \hat{c}_{i \sigma}(t)\right\rangle$ (with indices in the bound sector) has been derived elsewhere [26] and reads $\dot{\rho}=-i\left[h_{\mathrm{HF}}[\rho], \rho\right]-\mathcal{I}[\rho]-\mathcal{I}^{\dagger}[\rho]$. Here the HF Hamiltonian $h_{\mathrm{HF}}(t) \equiv h+V_{\mathrm{HF}}(t)+\mathbf{E}(t) \cdot \mathbf{d}$ is a functional of $\rho$ through the HF potential $V_{\mathrm{HF}, i j}(t)=\sum_{m n} \rho_{n m}(t) w_{i m n j}$, with $w_{i m n j} \equiv 2 v_{i m n j}-v_{i m j n}$. Dynamical correlation and ionization processes are described by the generalized collision integral

$$
\mathcal{I}(t)=\int_{0}^{t} d \bar{t}\left[\Sigma^{>}(t, \bar{t}) G^{<}(\bar{t}, t)-\Sigma^{<}(t, \bar{t}) G^{>}(\bar{t}, t)\right],
$$

where $\Sigma^{\lessgtr} \equiv \Sigma_{\mathrm{c}}^{\lessgtr}+\Sigma_{\text {ion }}^{\lessgtr}$ is the sum of the lesser or greater correlation $\left(\Sigma_{\mathrm{c}}\right)$ and ionization $\left(\Sigma_{\text {ion }}\right)$ self-energies. Both are timenonlocal functionals of $\rho$ through the generalized KadanoffBaym ansatz [29] (GKBA) [see Supplemental Material (SM) for details [30]]. Figure 1(b) illustrates the diagrammatic representation of $\Sigma_{\mathrm{c}}$ in the second-Born (2B) approximation and $\Sigma_{\text {ion }}$. The computational cost of these NEGF calculations scales like $N_{t}^{2} N_{\text {bound }}^{\mathfrak{p}}$ where $N_{t}$ is the number of time steps, $N_{\text {bound }}$ is the number of HF bound states, and the power $3 \leqslant$ $\mathfrak{p} \leqslant 5$ depends on how sparse $v_{i j m n}$ is. Real-time simulations of, e.g., organic or biologically relevant molecules can easily be carried out up to 30-40 fs [28].

The inclusion of Auger scattering processes leads to a coupling between the density matrix $\rho(t)$ and the occupations $f_{\mu}(t)=\left\langle\hat{c}_{\mu \sigma}^{\dagger}(t) \hat{c}_{\mu \sigma}(t)\right\rangle$ of the continuum states. For these quantities we have derived (see SM) the following coupled system of NEGF equations of motion:

$$
\begin{aligned}
\dot{\rho} & =-i\left[h_{\mathrm{HF}}[\rho], \rho\right]-\mathcal{I}[\rho, f]-\mathcal{I}^{\dagger}[\rho, f], \\
\dot{f}_{\mu} & =-\mathcal{J}_{\mu}[\rho, f]-\mathcal{J}_{\mu}^{*}[\rho, f] .
\end{aligned}
$$

The generalized collision integral $\mathcal{I}[\rho, f]$ is defined as in Eq. (3) but $\Sigma[\rho] \rightarrow \Sigma[\rho]+\Sigma_{\text {Auger }}[\rho, f]$. The Auger selfenergy is calculated from the second-order (in $v^{A}$ ) diagrams, in accordance with Refs. [31,32], and reads

$$
\begin{aligned}
& \sum_{\text {Auger }, i j}^{\lessgtr}(t, \bar{t})=\sum_{m n p q} \sum_{\mu} G_{m n}^{\lessgtr}(t, \bar{t}) \\
& \quad \times\left[G_{\mu}^{\lessgtr}(t, \bar{t}) G_{p q}^{\gtrless}(\bar{t}, t)\left(v_{i q m \mu}^{A} w_{\mu n p j}^{A}+v_{i q \mu m}^{A} w_{n \mu p j}^{A}\right)\right. \\
& \left.\quad+G_{p q}^{\lessgtr}(t, \bar{t}) G_{\mu}^{\gtrless}(\bar{t}, t) v_{i \mu p m}^{A} w_{n q \mu j}^{A}\right],
\end{aligned}
$$

where we neglected the off-diagonal elements of the continuum Green's function, i.e., $G_{\mu \nu}^{\lessgtr}=\delta_{\mu \nu} G_{\mu}^{\lessgtr}$. As we shall demonstrate, this approximation is remarkably accurate. Through the GKBA, $\Sigma_{\text {Auger }}$ is a time-nonlocal functional of $\rho$ and $f_{\mu}$. Finally, the collision integral $\mathcal{J}_{\mu}$ reads

$$
\mathcal{J}_{\mu}(t)=\int_{0}^{t} d \bar{t}\left[K_{\mu \mu}^{>}(t, \bar{t}) f_{\mu}^{<}(\bar{t})+K_{\mu \mu}^{<}(t, \bar{t}) f_{\mu}^{>}(\bar{t})\right],
$$

where the kernel

$$
\begin{aligned}
K_{\mu \nu}^{\lessgtr}(t, \bar{t})= & i \sum_{m n p q s r} v_{\mu r p m}^{A} w_{n q s v}^{A} \\
& \times G_{m n}^{\lessgtr}(t, \bar{t}) G_{p q}^{\lessgtr}(t, \bar{t}) G_{s r}^{\gtrless}(\bar{t}, t) e^{-i \epsilon_{v}(\bar{t}-t)}
\end{aligned}
$$

is a time-nonlocal functional of $\rho$ only. Equations (4), together with the definitions that follow it, constitute the first (methodological) result of this Rapid Communication. The implementation of Eqs. (4) does not alter the quadratic scaling with $N_{t}$. The scaling with the number of basis functions changes from $N_{\text {bound }}^{\mathfrak{p}}$ to $\max \left[N_{\text {bound }}^{\mathfrak{p}}, N_{\text {bound }}^{\mathfrak{q}} N_{\text {cont }}\right]$ where $N_{\text {cont }}$ is the number of continuum states and $2 \leqslant \mathfrak{q} \leqslant 4$. Therefore, the proposed equations can be used to simulate a large class of molecules of current interest.

Assessment of NEGF approach. To demonstrate the reliability of the coupled NEGF Eqs. (4) we consider a 1D atom with soft Coulomb interactions. On the grid points $x_{n}=$ $n a$ with $|n|<N_{\text {grid }} / 2$, the single-particle Hamiltonian reads $h\left(x_{n}, x_{m}\right)=\delta_{n, m}\left[2 \kappa+V_{\mathrm{n}}\left(x_{n}\right)\right]-\delta_{|n-m|, 1} \kappa$, where the nuclear 


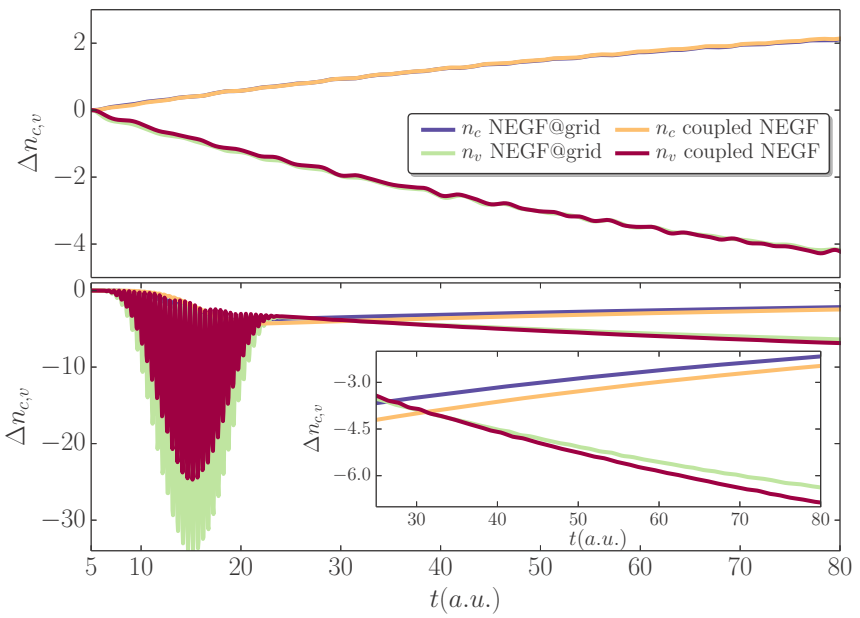

FIG. 2. Variation of the TD occupations (per spin) $n_{c}(t)$ [core, increasing blue (dark-gray) and orange (light-gray) curves] and $n_{v}(t)$ [valence, decreasing green (light-gray) and red (dark-gray) curves] calculated using NEGF@grid and coupled NEGF Eqs. (4) for the sudden creation of a core hole (top) and the action of a laser pulse (bottom). The inset shows a magnification of $n_{c}(t)$ and $n_{v}(t)$ after the end of the pulse. Vertical axes have been scaled up by a factor $10^{2}$.

potential $V_{\mathrm{n}}(x)=U_{\mathrm{en}} / \sqrt{x^{2}+a^{2}}$ for $|x| \leqslant R$ and $V_{\mathrm{n}}(x)=0$ otherwise. Electrons interact only in a box of length $2 R$ centered around zero through $v\left(x, x^{\prime}\right)=Z U_{\text {ee }} / \sqrt{\left(x-x^{\prime}\right)^{2}+a^{2}}$. The coupling to an external laser pulse is accounted for by adding $\delta_{n m} x_{n} E(t)$ to $h\left(x_{n}, x_{m}\right)$.

We take $N_{\text {grid }}=400$ and (henceforth all quantities are expressed in atomic units) $a=0.5, \kappa=2, Z=4, U_{\text {en }}=2$, $U_{\mathrm{ee}}=U_{\mathrm{en}} / 2$, and $R=10 a$. With four electrons the HF spectrum has $N_{\text {sys }}=5$ bound states (per spin) and $N_{\text {cont }}=N_{\text {grid }}-$ $N_{\text {sys }}$ continuum states. The occupied levels have energy $\epsilon_{c}=$ -4.33 (core) and $\epsilon_{v}=-1.65$ (valence). The HF states are used to construct the Hamiltonian in Eqs. (1) and (2). The results obtained by solving the coupled NEGF Eqs. (4) (where $\rho$ is a $N_{\text {sys }} \times N_{\text {sys }}$ matrix and $f$ is a $N_{\text {cont }}$-dimensional vector) are benchmarked against NEGF calculations on the full grid (NEGF@grid). NEGF@grid simulations are performed by solving the original equation [26] $\dot{\rho}=-i\left[h_{\mathrm{HF}}[\rho],\right]-\mathcal{I}[\rho]-$ $\mathcal{I}^{\dagger}[\rho]$ where all quantities are $N_{\text {grid }} \times N_{\text {grid }}$ matrices in the $x_{n}$ basis and $\mathcal{I}$ is given by Eq. (3) with $\Sigma=\Sigma_{\mathrm{c}}$ (see SM for details). By construction, NEGF@grid simulations include the off-diagonal elements $h_{i \mu}, h_{\mu \mu^{\prime}}, \mathbf{d}_{\mu \mu^{\prime}}$ and all Coulomb integrals with two or more indices in the continuum. Notice that NEGF@ grid scales cubically with $N_{\text {cont }}$ and it is therefore not exportable to large systems.

In Fig. 2 we show the time-dependent (TD) occupation (per spin) of the core, $n_{c}$, and valence, $n_{v}$, levels. In the top panel we suddenly remove $4 \%$ of charge from the core, hence $\rho_{c c} \rightarrow$ $\rho_{c c}-n_{h}$ with $n_{h}=0.04$, and let the system evolve without external fields. In the bottom panel the equilibrium system is driven by the external pulse

$$
E(t)=E_{0} \sin ^{2}\left(\frac{\pi t}{T}\right) \sin (\Omega t)
$$

with central frequency $\Omega=6.2$, active from $t=0$ until $t=$ $T=20$. The frequency is large enough for the energy of the

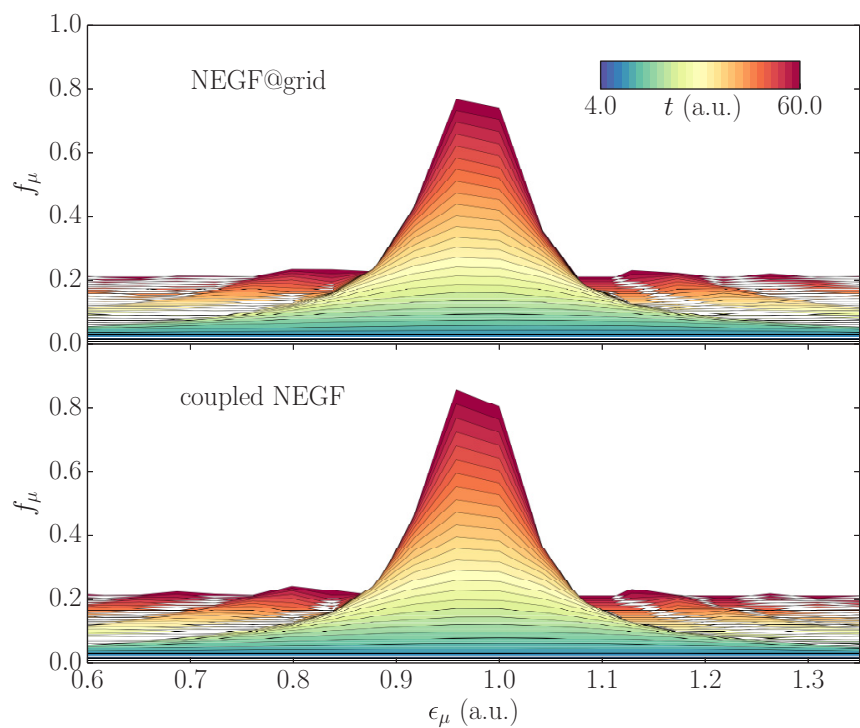

FIG. 3. Time-dependent occupations $f_{\mu}(t)$ of the continuum states versus their energy $\epsilon_{\mu}$ after the sudden creation of a core hole. The results are obtained from the solution of the NEGF@ grid equation (top) and coupled NEGF Eqs. (4) (bottom). In both cases the maximum occurs at $\epsilon_{\mu}=\epsilon_{\text {Auger }}$. Vertical axes have been scaled up by a factor $10^{2}$.

photoelectron not to overlap with the energy of the Auger electron. The intensity has been chosen to have the same amount of expelled charge as in the case of the sudden removal: $E_{0}=2.0$ for NEGF@grid and $E_{0}=1.5$ for the coupled NEGF Eqs. (4) - the difference in the value of $E_{0}$ is due to the neglect of the dipole elements $d_{\mu \mu^{\prime}}$ in Eq. (2). The results perfectly agree in the top panel, whereas only a minor discrepancy is observed in the bottom panel. In both type of simulations the Auger decay slightly depends on how the core hole is created. In fact, the laser pulse is also responsible for expelling charge from the valence level, thereby hindering the refilling of the core. The core-hole lifetime agrees well with the inverse linewidth function $\Gamma\left(\epsilon_{\text {Auger }}\right)=$ $2 \pi \sum_{\mu}\left|v_{c \mu v v}\right|^{2} \delta\left(\epsilon_{\text {Auger }}-\epsilon_{\mu}\right) \simeq 10^{-2}$ in all cases. It is worth emphasizing that no time-local approximation of $\Sigma_{\text {Auger }}$ would yield the behavior $n_{c}(t)=1-n_{h} e^{-\Gamma t}$. We performed TD HF simulations both in the grid basis and by solving Eqs. (4) with $\mathcal{J}_{\mu}=\Sigma_{\mathrm{c}}=\Sigma_{\text {Auger }}=0$, and found that $n_{c}(t)$ remains essentially constant (not shown). This is consistent with similar findings obtained in TDDFT using adiabatic xc potentials [19].

After the sudden creation of a core hole the electronic density populates the continuum states $\varphi_{\mu}$. In Fig. 3 we show the corresponding time-dependent occupations $f_{\mu}(t)$ versus their energy $\epsilon_{\mu}$. Again simulations have been performed using NEGF@grid (top panel) and the coupled NEGF Eqs. (4) (bottom panel). As time passes the total expelled charge increases and $f_{\mu}(t)$ gets peaked at the Auger energy $\epsilon_{\text {Auger }}=2 \epsilon_{v}-\epsilon_{c} \simeq$ 1. The final profile of the peak has a width $\Gamma \equiv \Gamma\left(\epsilon_{\text {Auger }}\right)$, independently of how the core hole is created (suddenly or due to a laser pulse). On the contrary, the photoelectron peak attains a width $\sim 2 \pi / T$ immediately after the end of the pulse (see animation continuum_occupations.mp4 in the $\mathrm{SM})$. We also observe that the exact energy of the Auger 
electron $\epsilon_{\text {Auger }}^{\text {exact }}=2 \epsilon_{v}-\epsilon_{c}-v_{v v v v}$ is not within reach of the second-order approximation in Eq. (5): the shift $v_{v v v v}$ (due to the valence-valence repulsion) would require a $T$-matrix treatment $[33,34]$. However, such shift has only a minor impact on the internal dynamics of 3D systems like, e.g., organic molecules, since the repulsion between two valence holes is typically less than $1 \mathrm{eV}$.

Auger wave-packet reconstruction. We now use the coupled NEGF Eqs. (4) to study the 1D atom on larger boxes (hence one- and two-electron integrals are calculated from HF states that spread over a large number of grid points). The output has been postprocessed to reconstruct the density of the Auger wave packet according to $n_{\text {Auger }}(x, t)=$ $\sum_{\mu \nu} \varphi_{\mu}^{*}(x) f_{\mu \nu}(t) \varphi_{\nu}(x)$, where $f_{\mu \nu}(t)=\left\langle\hat{c}_{\nu \sigma}^{\dagger}(t) \hat{c}_{\mu \sigma}(t)\right\rangle$ is the off-diagonal density matrix in the continuum sector. The latter is obtained by integrating the NEGF equation of motion (see $\mathrm{SM}$ for the derivation)

$$
\dot{f}_{\mu \nu}=-i\left(\epsilon_{\mu}-\epsilon_{\nu}\right) f_{\mu \nu}-\mathcal{J}_{\mu \nu}[\rho, f]-\mathcal{J}_{\nu \mu}^{*}[\rho, f],
$$

where $\mathcal{J}_{\mu \nu}$ is given by the right-hand side of Eq. (6) after the replacement $K_{\mu \mu}^{\lessgtr}(t, \bar{t}) f_{\mu}^{\gtrless}(\bar{t}) \rightarrow K_{\mu \nu}^{\lessgtr}(t, \bar{t}) f_{\nu}^{\gtrless}(\bar{t})$.

In Fig. 4 we display the Auger wave packet for $N_{\text {grid }}=1600$ grid points. In the top panel the core hole is suddenly created (see also animation Auger_wavepacket.mp4 in the SM), whereas in the middle panel the atom is driven by the ionizing laser of Eq. (8). The first observation is that the wave front depends on the perturbation (sudden creation or laser), being steeper the shorter it takes to create the hole. The wave packet moves rightward at the expected speed $v=\partial \epsilon / \partial p \simeq 2 \sqrt{\kappa \epsilon_{\text {Auger }}}=2.2$ and its length is approximately $v / \Gamma$ far away from the nucleus. Interestingly, the tail of the wave packet exhibits spatial ripples that tend to accumulate nearby the origin. The amplitude of the ripples depends on the perturbation (sudden creation or laser), whereas their spacing is an intrinsic feature. In the bottom panel of Fig. 4 we show the period $T_{r}$ of the ripples, i.e., the elapsing time between two consecutive maxima of $n_{\text {Auger }}\left(x_{0}, t\right)$, at the interface $x_{0}=30 a$, versus the number of periods. We present results for three different values of range and strengths of the Coulomb force $\left(R, U_{\mathrm{en}}, U_{\mathrm{ee}}\right)=$ $(10 a, 2,1),(100 a, 2.6,2.08)$, and $(10 a, 2.7,2.025) \quad$ yielding Auger energies $\epsilon_{\text {Auger }}=1.02,1.76$, and 2.66, respectively. In all cases we find that $T_{r}$ attains a finite limit given by

$$
T_{r}=2 \pi / \epsilon_{\text {Auger }} .
$$

The occurrence of ripples and the intrinsic period $T_{r}$ is not an artifact of the self-energy approximation. These features as well as the overall shape of the Auger wave packet are indeed confirmed by Configuration Interaction (CI) calculations. Starting at time $t=0$ with the photoexcited state $\left|\Phi_{\mathrm{x}}\right\rangle=\hat{c}_{c \uparrow}^{\dagger} \hat{c}_{v \downarrow}^{\dagger} \hat{c}_{v \uparrow}^{\dagger}|0\rangle$ and evolving with the Hamiltonian in Eq. (1) one finds $n_{\text {Auger }}(x, t)=\left|\varphi_{\text {Auger }}(x, t)\right|^{2}$ with $\varphi_{\text {Auger }}(x, t)=\sum_{\mu} a_{\mu}(t) \varphi_{\mu}(x)$ and

$$
a_{\mu}(t) \simeq-v_{c \mu v v} e^{-i E_{\mu} t} \frac{e^{i\left(\epsilon_{\mu}-\epsilon_{\text {Auger }}+i \Gamma / 2\right) t}-1}{\epsilon_{\mu}-\epsilon_{\text {Auger }}+i \Gamma / 2} .
$$

The CI Auger wave packet is in excellent agreement with NEGF (see the SM). In the SM we further show that the ripples
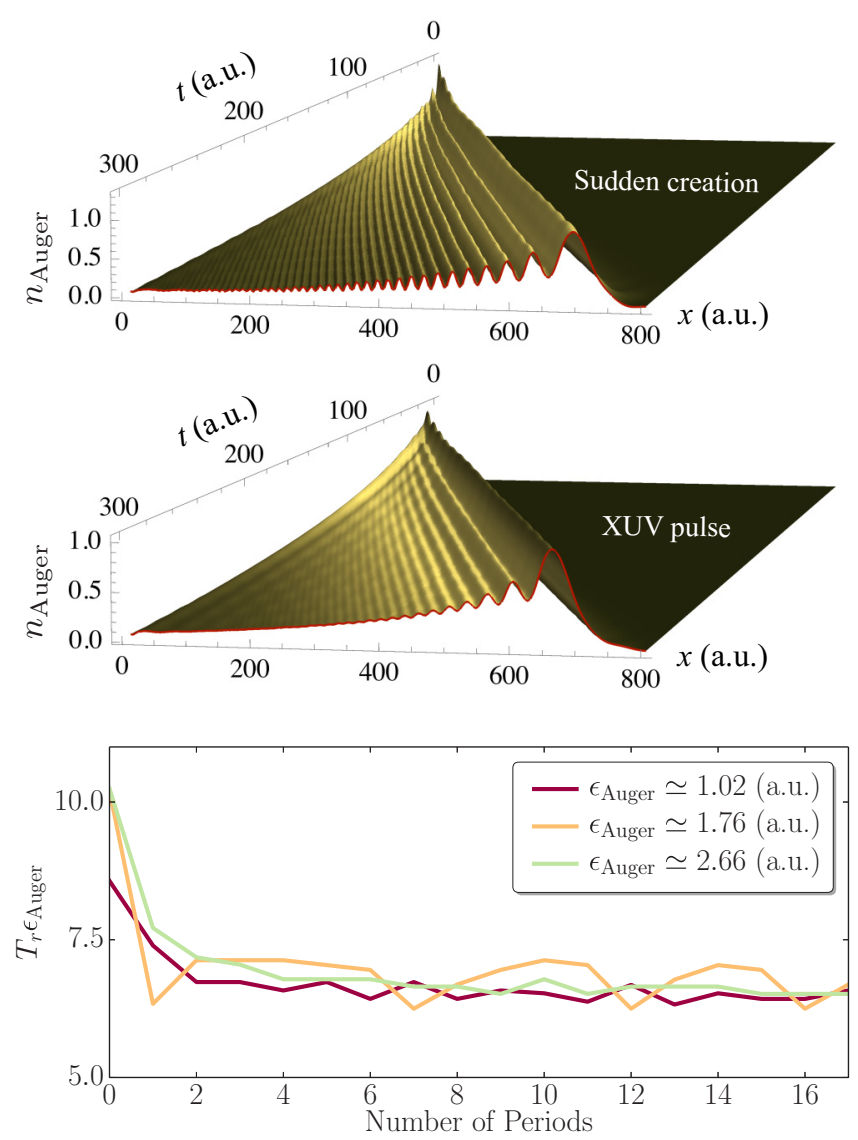

FIG. 4. Snapshots of the density of the Auger wave packet after the sudden creation of a core hole (top) and the action of a laser pulse (middle). The bottom panel shows the period of the ripples at an interface versus the number of periods for three different values of range and strengths of the Coulomb force (see main text) yielding Auger energies $\epsilon_{\text {Auger }}=1.02$ [red (dark-gray)], 1.76 [yellow (gray)], and 2.66 [green (light-gray)].

occur even in two or three dimensions and, therefore, they are a fingerprint of the Auger electron.

To summarize, we have included Auger decays in a firstprinciples NEGF approach to simulate the UCM dynamics of molecules driven by attosecond pulses. The computational effort is comparable to that of previous NEGF implementations [26-28], thereby allowing for studying systems with tens of active electrons up to tens of femtoseconds. Benchmarks in $1 \mathrm{D}$ atoms demonstrate that both qualitative and quantitative aspects are well captured. We also predict a highly asymmetric profile of the Auger wave packet with a spatial extension of the order $v / \Gamma$ and superimposed ripples with temporal period $T_{r}=2 \pi / \epsilon_{\text {Auger }}$.

Although the fundamental equations have been derived for finite systems, the proposed NEGF approach can be extended to deal with periodic systems too. In this context the equation of motion for the single-particle density matrix opens the possibility to develop current-density functional theories that include dissipation and thermalization.

Acknowledgments. G.S. and E.P. acknowledge EC funding through the RISE Co-ExAN (Grant No. GA644076). E.P. also acknowledges funding from the European Union project MaX 
Materials design at the eXascale H2020-EINFRA-2015-1, Grant Agreement No. 676598 and Nanoscience Foundries and Fine Analysis-Europe H2020-INFRAIA-2014-2015, Grant Agreement No. 654360. F.C. and A.R. acknowledge financial support from the European Research Council (ERC-2015AdG-694097), Grupos Consolidados (IT578-13), and European Union Horizon 2020 program under Grant Agreement No. 676580 (NOMAD).
[1] F. Calegari, D. Ayuso, A. Trabattoni, L. Belshaw, S. De Camillis, S. Anumula, F. Frassetto, L. Poletto, A. Palacios, P. Decleva et al., Science 346, 336 (2014).

[2] M. Uiberacker, T. Uphues, M. Schultze, A. J. Verhoef, V. Yakovlev, M. F. Kling, J. Rauschenberger, N. M. Kabachnik, H. Schröder, M. Lezius et al., Nature (London) 446, 627 (2007).

[3] A. I. Kuleff and L. S. Cederbaum, J. Phys. B 47, 124002 (2014).

[4] A. I. Kuleff, N. V. Kryzhevoi, M. Pernpointner, and L. S. Cederbaum, Phys. Rev. Lett. 117, 093002 (2016).

[5] J. Breidbach and L. S. Cederbaum, J. Chem. Phys. 118, 3983 (2003).

[6] N. V. Golubev and A. I. Kuleff, Phys. Rev. A 91, 051401 (2015).

[7] K. Nagaya, H. Iwayama, A. Sugishima, Y. Ohmasa, and M. Yao, Appl. Phys. Lett. 96, 233101 (2010).

[8] M. Kutzner, V. Maycock, J. Thorarinson, E. Pannwitz, and J. A. Robertson, Phys. Rev. A 66, 042715 (2002).

[9] T. Uphues, M. Schultze, M. F. Kling, M. Uiberacker, S. Hendel, U. Heinzmann, N. M. Kabachnik, and M. Drescher, New J. Phys. 10, 025009 (2008).

[10] M. Drescher, M. Hentschel, R. Kienberger, M. Uiberacker, V. Yakovlev, A. Scrinzi, Th. Westerwalbesloh, U. Kleineberg, U. Heinzmann, and F. Krausz, Nature (London) 419, 803 (2002).

[11] S. Zherebtsov, A. Wirth, T. Uphues, I. Znakovskaya, O. Herrwerth, J. Gagnon, M. Korbman, V. S. Yakovlev, M. Vrakking, M. Drescher et al., J. Phys. B 44, 105601 (2011).

[12] J. M. Schins, P. Breger, P. Agostini, R. C. Constantinescu, H. G. Muller, G. Grillon, A. Antonetti, and A. Mysyrowicz, Phys. Rev. Lett. 73, 2180 (1994).

[13] O. Smirnova, V. S. Yakovlev, and A. Scrinzi, Phys. Rev. Lett. 91, 253001 (2003).

[14] A. K. Kazansky, I. P. Sazhina, and N. M. Kabachnik, J. Phys. B 42, 245601 (2009).

[15] A. K. Kazansky, I. P. Sazhina, and N. M. Kabachnik, J. Phys. B 44, 215601 (2011).

[16] E. Runge and E. K. U. Gross, Phys. Rev. Lett. 52, 997 (1984).

[17] C. Ullrich, Time-Dependent Density-Functional Theory (Oxford University Press, Oxford, 2012).
[18] N. T. Maitra, J. Chem. Phys. 144, 220901 (2016).

[19] C. S. Cucinotta, D. Hughes, and P. Ballone, Phys. Rev. B 86, 045114 (2012).

[20] The inadequacy of the adiabatic approximation is easily understandable. The Auger electron adds to the main quasiparticle peak a secondary peak in the spectral function of the parent cation. An adiabatic approximation can, at most, renormalize the main quasiparticle peak.

[21] L. P. Kadanoff and G. A. Baym, Quantum Statistical Mechanics: Green's Function Methods in Equilibrium and Nonequilibirum Problems (Benjamin, New York, 1962).

[22] G. Stefanucci and R. van Leeuwen, Nonequilibrium ManyBody Theory of Quantum Systems: A Modern Introduction (Cambridge University Press, Cambridge, 2013).

[23] P. Myöhänen, A. Stan, G. Stefanucci, and R. van Leeuwen, Phys. Rev. B 80, 115107 (2009).

[24] P. Myöhänen, A. Stan, G. Stefanucci, and R. van Leeuwen, Europhys. Lett. 84, 67001 (2008).

[25] S. Latini, E. Perfetto, A.-M. Uimonen, R. van Leeuwen, and G. Stefanucci, Phys. Rev. B 89, 075306 (2014).

[26] E. Perfetto, A.-M. Uimonen, R. van Leeuwen, and G. Stefanucci, Phys. Rev. A 92, 033419 (2015).

[27] E. V. Boström, A. Mikkelsen, C. Verdozzi, E. Perfetto, and G. Stefanucci, Nano Lett. 18, 785 (2018).

[28] E. Perfetto, D. Sangalli, A. Marini, and G. Stefanucci, J. Phys. Chem. Lett. 9, 1353 (2018).

[29] P. Lipavský, V. Špička, and B. Velický, Phys. Rev. B 34, 6933 (1986).

[30] See Supplemental Material at http://link.aps.org/supplemental/ 10.1103/PhysRevA.97.061401 for the derivation of the theoretical framework as well as for the animations of the continuum occupations and Auger wave packet in real space.

[31] C.-O. Almbladh, A. L. Morales, and G. Grossmann, Phys. Rev. B 39, 3489 (1989).

[32] C.-O. Almbladh and A. L. Morales, Phys. Rev. B 39, 3503 (1989).

[33] M. Cini, Solid State Commun. 88, 1101 (1993).

[34] G. A. Sawatzky, Phys. Rev. Lett. 39, 504 (1977). 